\title{
Gaining Insight and Taking Action: Engaging Adults with Persistent Pain and Opioid Use Disorder in an Online Pain Self-Management Program
}

\author{
Marian Wilson, PhD, MPH, RN \\ Washington State University College of Nursing \\ marian.wilson@wsu.edu
}

\author{
Michele R. Shaw, PhD, RN \\ Washington State University College of Nursing \\ michele_shaw@wsu.edu
}

\begin{abstract}
The primary purpose of this study was to evaluate participants' perceptions after engaging in an 8-week online pain self-management program. Participants $(N=31)$ were adults enrolled in a medication-assisted outpatient opioid treatment program who had coexisting opioid substance use disorder and persistent pain. Data were collected via secure online surveys and content analysis methods were used to analyze text from open-ended questions. Two themes were identified describing benefits of the program: gaining insight and taking action. Two themes described how participants would like to improve program experiences: feeling overwhelmed and ease of use. Survey data were also examined for relationships between level of program engagement, pain relief, and substance use to explore potential barriers to program use. Poorly managed pain and illicit drug use were associated with reduced program use $(p<0.05)$. Understanding preferences and barriers can assist adoption of online programs for people with comorbid pain and substance use disorder.
\end{abstract}

\section{Introduction}

Opioid substance use disorder (SUD) has reached epidemic proportions in the United States (U.S) with approximately 3.8 million Americans aged 12 or older reporting current misuse of prescription pain relievers [1]. Opioid overdose deaths nearly tripled during the past two decades and are now the second leading cause of accidental death in the U.S., with nearly 115 deaths per day [2]. Chronic pain has been recognized as an important motivator leading individuals to misuse opioids (e.g. hoarding or non-prescribed use) [3]. More than half of those with chronic pain describe it as "unbearable" or "excruciating" [4].

While much energy has gone towards addressing SUDs, less attention has been paid to the fact that persistent (or chronic) pain is a comorbid condition for many receiving opioid SUD treatment. Estimates are that between $27-80 \%$ of adults enrolled in an opioid SUD treatment program have co-existing persistent pain [5]. Yet, pain is often managed inadequately or inappropriately among people receiving opioid SUD treatment [6].

It is unknown how these undertreated symptoms may contribute to SUD treatment success and affect quality of life. Therefore, we sought to test a pain selfmanagement program, the online "Chronic Pain Management Program," that has previously been found to be helpful for people with painful conditions, yet has never been tested among people with coexisting opioid SUD [7,8]. The present study was nested within a randomized controlled trial (RCT) that has been reported on previously for efficacy $[9,10]$. Because program engagement was subpar, the present study builds on what was previously learned with the primary research question being: (1) What are participants' perspectives after engaging in an online pain self-management program? A secondary aim of the present study was to explore relationships between clinically pertinent factors and program use and answer the secondary research question: (2) Are any clinical variables significantly associated with program engagement? Our present study examined unexplored data with the specific objective of determining how to improve the online program uptake by participants. This information can provide insight on maximizing the program's usefulness for our population of interest, and also may yield information to assist in future technology developments.

\section{Background}

\subsection{Persistent pain and opioid use}

An estimated 25 million (11\%) United States (U.S.) adults experience persistent (or chronic) pain, defined as any pain lasting $\geq 3$ months that does not respond to treatment [11]. Effective pain treatment 
approaches include behavioral and cognitive therapies that assist people in how they interpret, think about, and respond to painful stimuli $[12,13]$. These approaches have been effectively delivered through online programs $[7,8,14]$, yet for many people with pain, such psychologically-based care is not routinely offered as a standard treatment approach [15].

Particularly for people in opioid SUD treatment, access to multidisciplinary and/or nonpharmacological treatment for pain can be elusive. As few as $13 \%$ of people in treatment for opioid SUD may receive any treatment at all for their persistent pain [5]. Yet, when they do receive pain care, their pain can be substantially improved [5]. An important long-term treatment option for opioid SUD is medicationassisted therapy (MAT) to reduce cravings and prevent illicit drug use. MAT programs use a synthetic opioid, methadone or buprenorphine, to treat opioid SUD. In the U.S., enrollment in MAT has been steadily climbing [16]. Because many opioid overdoses are linked to methadone or other opioids used in MAT [2], it is essential that opioid-sparing tactics are available to reduce overdose risks while simultaneously addressing pain [17].

Non-pharmacological methods of pain management could be an important strategy to reduce opioid overdose deaths, particularly for people who are already consuming opioids as part of MAT for SUD treatment. Opioid overdose deaths often occur as an unintended consequence of legitimate opioid prescribing practices [18]. Respiratory depression is the main hazard of opioid use [19]. People who are in opioid SUD treatment and receive opioid replacement therapies through MAT compound their risks of overdose if they consume additional opioids for pain relief.

The need to increase non-pharmacological options for all people with pain has been recognized by the U.S. Department of Health and Human Services' National Pain Strategy [20]. The need to improve outcomes for the millions of adults living with SUD is also apparent. A review by Eyler (2013) of 109 articles on pain management for patients in MAT concluded that treatment for this population is complicated by multiple factors, including heightened sensitivity to pain (hyperalgesia), high opioid tolerance, crosstolerance to pain medicines, and illicit substance use [21]. It should not be underestimated how persistent pain can impact quality of life and negatively affect SUD treatment.

\subsection{Self-management interventions}

Self-management is one of the most effective and well-studied behavioral treatments for increasing one's ability to manage chronic conditions [20,22]. Programs are intended to assist people in mastering the tasks needed to live with a chronic condition by increasing confidence, or self-efficacy, in one's ability to cope with health symptoms [20]. Pain selfmanagement interventions are viewed as an essential component of evidence-based clinical practice guidelines for chronic pain, although no single program has been adopted and widely-disseminated [20]. Online and face-to-face self-management interventions have demonstrated improved outcomes in small, specific populations of patients who suffer with a variety of painful conditions, such as patients with fibromyalgia, headaches, and arthritis [13,22]. How to engage the general population of people with persistent pain is unclear, and even less delineated is how to engage people with co-existing opioid SUD.

Innovations have been developed using technology to deliver health resources or health care via electronic means (E-health). E-health offers one possible means of access to self-management programming for people with chronic pain and SUD. The E-health program used in the present study, the Chronic Pain Management Program (CPMP), was created by psychologists who are pain researchers. The CPMP is available to the public online with a paid subscription (approximately \$25 U.S. dollars per month). The CPMP is a self-directed, self-paced Internet-based self-management program intended for a general population of people with persistent non-cancer pain. The program targets cognitive, emotional, behavioral and social pain determinants with documented efficacy in improving symptoms for people on opioid therapy [7]. Our pilot work testing the CPMP among people in MAT found engagement was less than desired with only $64.5 \%$ of participants $(\mathrm{N}=31)$ engaging in available online content $[9,10]$. Reduced symptom burdens were noted among those who did use the program in pain severity, pain interference, and depressive symptoms (10). Opioid misuse was reported as reduced for those who engaged in the program content as well (10) and general satisfaction with the program was high $(9,10)$. Therefore, in the present study we seek to understand more about barriers and facilitators to program engagement in an effort to maximize the program's future usefulness.

The main lessons provided by the CPMP map onto four modules that can usually be completed across 8weeks and include: Thinking Better, Feeling Better, Doing More, and Relating Better. More description is provided on the program website 
https://pain.goalistics.com [7]. Learning modules include didactic materials and interactive activities. For example, the Thinking Better module asks participants to recognize negative thinking patterns and to stop, evaluate, and redirect self-defeating thoughts. Feeling Better guides participants through relaxation exercises and builds awareness of emotional triggers. Doing More demonstrates fitness exercises and teaches pacing activities. Relating Better assists in building a helpful support system and scheduling social activities. At the end of each activity, participants are asked to assign a helpfulness rating using a 1-to-5 star rating where 1 star = "not at all helpful" and 5 stars = "extremely helpful." Some activities are to be completed off-line, such as physical exercises, relaxation, or self-monitoring behaviors.

Prior research demonstrated the CPMP's ability to decrease pain severity, pain-related interference, perceived disability, depression, and pain-induced fear among participants recruited from the Internet [7]. In our previous trial of the CPMP among adults with an opioid prescription for persistent pain, the main findings were that $20.9 \%$ of CPMP users compared to $6.8 \%$ of control group participants reported decreasing or discontinuing their opioid medication [8]. Treatment group participants reported significantly greater decreases in opioid misuse, increases in pain self-efficacy, and a significantly greater proportion had a clinically significant decrease (i.e. $\geq 2$ points) in pain intensity (18\% vs. 6\%) [8]. Further evidence links improvements in self-efficacy to improvements in substance use treatment [23]. Thus, we believed the Ehealth program could be beneficial for adults with pain and a co-existing SUD. Our present analysis is intended to assist in understanding what enhancements may be needed to provide maximum benefit to this complex population.

\section{Methods}

For our primary aim, Aim 1, regarding participant perspectives after online program engagement, qualitative descriptive methodology using content analysis methods was selected to describe experiences and identify common themes [24,25]. Qualitative description is used when the goal of the research is to summarize descriptions of events or experiences in order to depict the perspectives of the participants [24, 25]. Common themes are identified in qualitative description through content analysis methods to provide definitions and details of the most prominent ideas provided by the participants' responses [24,25]. This methodology compliments the purpose of the present study by allowing the participants of the online pain self-management program to give subjective, detailed input about the programs' usefulness.

A quantitative descriptive approach was followed for our secondary aim, Aim 2, to investigate if specified clinical factors were associated with program engagement. Correlations were calculated on minimally-structured numeric data [24] among previously unexamined variables including: (1) survey data on self-reported pain relief and substances used for pain relief, (2) electronic clinical record data on urine drug screens and daily opioid dose, and (3) the CPMP online activity records. These variables were chosen to evaluate whether pain, substance use, or prescribed medications might be related to online program engagement.

The parent randomized controlled study tested the CPMP by enrolling 60 U.S. adults who were prescribed opioid replacement therapy for opioid addiction and who had co-existing persistent pain. Quantitative efficacy data were analyzed and reported elsewhere [10]. A total of 111 potential participants were screened for the original study, 60 (54\%) were referred from the treatment clinic staff and 51 (45.9\%) were self-referred from advertisements posted in the clinic. Of those screened for eligibility, 51 were not enrolled, primarily due to not following up with the consent procedure $(n=37)$, while 7 were found ineligible and 7 declined to enroll. Of the 60 participants who consented to join the original RCT, 39 (17 treatment group, 22 control group) completed all study procedures over the 8-week study period (36.6\% attrition). The present study data is from baseline data from 31 of the original 60 participants who were randomized to the treatment arm testing the CPMP. Of those 31, 17 contributed text data to openended survey questions after trialing the CPMP; these text responses were used to address Aim 1. Statistical calculations for Aim 2 were performed on data from the full treatment group sample $(n=31)$.

Eligibility criteria included individuals 18 years of age or older who: (1) self-identified as having had a non-cancer chronic pain lasting for greater than 3 months; (2) were enrolled in a supervised opioid addiction treatment program and receiving opioid replacement therapy; (3) had email capability either at home or at a public setting (computers made available for use at the study sites); (4) had ability to read, speak and write in the English language; and (5) had ability to provide written informed consent. Exclusion criteria were chosen to limit confounding treatment effects and included: (1) medical or psychiatric condition that the principal or co-investigators determined would compromise safe study participation (such as behavioral issues or violations of clinic regulations); (2) pregnancy; and/or (3) currently enrolled in 
psychological counselling specifically for pain management.

\subsection{Data collection}

All procedures were reviewed and approved by the Institutional Review Board of the university sponsoring the study. Surveys were collected online using a secure survey site. Pertinent to Aim 1, openended survey questions were included in the qualitative analysis. The three items were presented at the end of the study in an online survey to evaluate participants' experiences with using the online pain self-management program: 1) "Did you find anything about this program especially useful? What would that be?" 2) "Is there anything you would change about this program if you could? What would that be?" and 3) "What else can you share about your experience participating in this program?"

For Aim 2, numeric data on clinical aspects were collected using several data sources: (1) a validated pain relief item from the Brief Pain Inventory instrument was answered on the baseline survey asking "In the last 24 hours, how much relief have pain treatments or medications provided?" with options ranging from $0 \%$ (no relief) to $100 \%$ (complete relief) [26]; (2) self-reported items that were developed by the research team instructed participants on the baseline survey to "Check off any substance you have used to help control your pain" listing 15 substances assessed for commonly in addictions trials, including alcohol, cannabis, nicotine, stimulants, sedatives, and heroin; (3) data from electronic clinical records were extracted by researchers on urine drug screen results and daily opioid dose (converted into morphine equivalency dose) during the 8-week study period; and, (4) program engagement data were collected using methods devised for prior online selfmanagement intervention studies [8-10] and calculated as a binary variable to represent level of engagement, where $0=$ no engagement vs. $1=$ at least partial engagement [evidenced by logging into the online program].

Ryan and Sawin's Individual and Family SelfManagement Theory (IFSMT) (2009) provided theoretical background to our study [27]. In the IFSMT, the individual or family assumes responsibility for self-management, and may include health care providers as collaborators. In this study, we expected many participants would not have regular access or experience with computers so we planned to use research assistants who were trained in the online self-management program to serve as collaborators as they guided participants through the program. Research staff assisted with computer access and skills. We also sought in Aim 2 to identify other variables that might support or inhibit online program use. These decisions were in alignment with the IFSMT which asks to consider unique physical, social and individual variables that may enhance or deter from achieving desired self-management program outcomes [27].

\subsection{Data analysis}

For Aim 1, content analysis methods as described by Schreier were used to analyze survey text data [28]. The data (responses to the three open-ended survey items) were de-identified and transferred into a word document table for analysis. Throughout the content analysis the researchers focused on identifying common themes in the text that were associated with participants' descriptions of the benefits and challenges of completing the online program. Following Schreier's qualitative content analysis methods, the authors initially read through the word document of the participants' responses separately and made notes describing their ideas for potential themes based on commonly identified statements throughout the data. The researchers then compared initial findings, reviewed initial summaries of overall impressions of the data, and identified agreed upon themes. The researchers returned back to the data and used the coding frame as a reference, continued on with analysis by further summarizing themes, continuing coding of data, along with contrasting similarities and differences among themes. The researchers compared individual analyses, revised themes and definitions, and compared identified quotes supporting the themes [28]. Reliability of the study was addressed by the process of having each researcher initially review and analyze the data prior to comparing consistency of agreement between the coders [28]. Consistency was high among the commonly identified themes and supporting quotes. Validity was also addressed by considering the applicability of the themes when compared to the participants' responses and the overall purpose of the study [28]. An audit trail was kept throughout the analysis process to document decisions and next steps. Quotations from the respondents were used to support the claims made.

For Aim 2, statistical analyses of numeric data were conducted in IBM SPSS Statistics version 21. Descriptive statistics included means and standard deviations (SD). Kendall's Tau correlations were calculated to determine if any significant relationships existed among the clinical variables of interest. The significance level was set at .05 (two-tailed). 


\section{Findings}

Participants were predominantly male (53\%), average age 44 years (SD 12), Caucasian (78\%), and $70 \%$ reported having education levels higher than a high school diploma. The most common pain diagnosis reported by participants was back or spine conditions $(45 \%)$, followed by nerve pain $(11.6 \%)$, surgical pain (8.3\%), fibromyalgia (6.6\%), and arthritis $(3.3 \%)$. The majority $(73 \%)$ reported their first use of opioids was from a legitimate painful event, while fewer reported first opioid use was for recreational purposes $(8 \%)$ or for psychological stress $(6 \%)$.

To address Aim 1, of 60 participants enrolled, all of the 17 who completing the 8 weeks in treatment group and their final posttest surveys contributed to the three open-ended items giving feedback on the online self-management program. Four themes were identified that provide description of the participants' perspectives about the online program. Themes describing the benefits of the program included: (1) gaining insight, and (2) taking action. Themes describing program challenges included: (4) feeling overwhelmed and (5) ease of use.

\subsection{Gaining insight}

The participants commonly reported that the online pain program provided new information and techniques that could be used to make positive shifts in the way they manage their pain. Several commented that participating in the program allowed them to gain knowledge, aid understanding, and see their situation in a new light. Gaining insight as a benefit of the program can be illustrated by the following quotes:

I thought the ways to not think about the pain were effective. I also felt that setting goals and doing the different activities really helped me understand my pain and helped me to not think about the pain and to overcome the pain while I did activities.

I did learn new stuff to try - the pacing activity. This was very helpful to me, because I'm that person who does it all wrong. But the pacing activity taught me how to do it right...I also learned from the relating better. I have been married for 30 years, and 1 learned a different way to relate to my husband, about my pain and what was helpful and what was not.
Participants shared comments about gaining general knowledge that "we can do something about the pain." This seemed to be a new insight for some. "Positive thinking" was acknowledged as a benefit along with being brought "to terms with your reality" via the program's focus on self-reflection. Gaining knowledge and information were commonly discussed such as one participant who said, "I will use the information to my benefit." Gaining insight was demonstrated with evidence of acceptance by the following quote:

I now know that I'm a full time pain management person.

\subsection{Taking action}

Participants reported a variety of skills and tools they received from the program that helped them take immediate steps to improve their situation. The online program provided physical exercises, a daily calendar for planning and tracking activities, and tips on pacing activities that were mentioned by participants as specific helpful components of the program that prompted new behaviors. Participants shared these examples:

The tracker was helpful, it allowed me to see how things affected my day, mood and pain level.

I have been using the program (sooo easy to use). I have not worked out in years. I AM NOW. Because I use the tools that are in this program. Is it hard? You bet it is, the difference is I can manage it, and get back to the business of life with Quality. For me it is life affecting in a positive manner. I can almost see myself again. And I know I'm well on my way to recovery with a job in the future.

Taking action was also evident in comments regarding how the program allowed participants to enjoy life again, schedule tasks, and engage in exercises. Other actions prompted by program use were mental in nature, such as positive self-talk participants reported including "Never give up" and "Visualize yourself in action." Adoption of coping skills was also noted and linked to daily actions as illustrated in the following comment:

The thought involved was put to immediate use with the coping skills used to carry on with my day. 


\subsection{Feeling overwhelmed}

The desire for a simpler structure that was less daunting was commonly described in the data. Participants expressed the need to have fewer tasks that could be tackled, particularly when starting the program. To some, it seemed when they began the program they were expected to do too many things at once. Participants expressed their challenges as illustrated in the following:

It was a bit overwhelming at first...It wasn't clear to me those activities were meant to span the entire 8 weeks...My next and only other complaint would be how extensive some tasks are. I feel like it would have been better if I was given a list of a few things to do each week instead of staring at what I thought were immensely long lists and having to decide on my own what to do.

Participants shared how they wanted more guidance when using the CPMP to lay out what to do first. Some thought the program was too long and had too many expectations that could not be met realistically. Two participant quotes illustrate this clearly:

I would start over if I could. I fell behind towards the beginning and unfortunately for me I never caught up.

Again, having the long lists and setting my own pace made it hard for me to stay focused. I would have done better with more defined expectations of what I should do each week.

\subsection{Ease of use}

Ease of use of the online program was another commonly identified theme. Participants desired reduced effort of navigation and some suggested a more formal orientation early on explaining how to use the online program. Only one reported technical difficulties due to no Internet access, and more often problems seemed related to making one's way around the program modules. Ease of use issues are expressed in the following examples, along with some improvement suggestions:

It was very confusing to use. The system just needs to be laid out better. Maybe like a step by step kind of set up. It was difficult to navigate.

I would make it more easier to use and understand. At the beginning make sure the participants understand where they have to go and what they need to do in order to get thru the various steps. Also get everyone together for a mandatory meeting and maybe go thru the steps with them. Do anything just to make this program more easy to navigate around the program.

Some aspects of the program intended to be helpful could be a source of frustration for participants as demonstrated by the following example:

Sometimes it was hard to check in every day. So maybe a weekly check in along with the daily. Then the person using the program can pick which one works for them. But that should also be after the person has been doing the program for awhile.

Participants overall seemed to desire an improved ease of use in order to successfully complete their assignments and fully engage in the online program. At least one participant did not find the program difficult, which may speak to the need to provide personalized support:

It was great, easy, simple checking in \& doing assignments. No problem.

\subsection{Clinical variables and engagement}

To address Aim 2, complete data were available from 31 participants randomized into the treatment group. On average, pain medications or treatments were said to have provided $31.3 \%$ pain relief to participants in the previous 24 hours. The most common substances reportedly used to provide pain relief are identified in Table 1.

\section{Table 1. Substances used to control pain}

\begin{tabular}{|l|c|c|}
\hline Type of substances & $\mathrm{n}$ & $\%$ \\
\hline Illegal use of prescription drugs & 21 & 67.7 \\
\hline Marijuana & 19 & 61.3 \\
\hline Street/illicit methadone & 18 & 58.1 \\
\hline Heroin & 17 & 54.8 \\
\hline Nicotine & 15 & 48.4 \\
\hline Benzodiazepines/Tranquilizers & 10 & 32.3 \\
\hline Alcohol & 9 & 29.0 \\
\hline Methamphetamines & 8 & 25.8 \\
\hline
\end{tabular}

Average morphine daily dose was $117 \mathrm{mg}$ per clinical electronic health records (SD 45.3). Positive urine drug screens for illicit drug use were identified on 17 of 31 treatment group members within the 8- 
week study duration (54.8\%). Drug screens were generally collected at random intervals every month as part of the partnering clinics' standard of care. Of the 31 treatment group members, 20 showed evidence of engaging in at least some online CPMP activities $(64.5 \%)$ with $5(16.1 \%)$ participants engaging in all major learning centers.

Significant positive relationships were found between online program engagement levels and (1) percentage of pain relief $(r t=0.42 ; p<0.01)$ and $(2)$ use of alcohol to control pain $(r t=0.36 ; p<0.05)$. Significant negative relationships were found between online program engagement and positive urine drug screen tests $(r t=-0.40 ; p<0.05)$. No significant relationships were found between program engagement and daily opioid dose or any other substances reportedly used for pain.

\section{Discussion}

Participant perspectives after online pain program engagement were summarized in four major emerging themes. The primary reported benefits were gaining insight and taking action. Reported challenges of program participation were described as feeling overwhelmed and ease of use. We can compare how the online program experience in this sample compared to our prior qualitative exploration of people with persistent pain and no diagnosed SUD [29]. Similar to ours, and other studies, the participants in the present study commonly reported that the online self-management intervention taught them skills that enabled them to adopt new behaviors or think differently, thereby gaining insight, about their situations [29-32]. Throughout the data, participants described how they had new knowledge and realizations. These insights often led to taking actions that were believed to be helpful new ways of managing or accepting pain, or its associated symptoms or sequalae.

One theme that has been noted in other qualitative studies of people with persistent pain involved in selfmanagement programs is that of feeling supported by others or not "being alone" in their suffering [30]. Shared experiences, emotional support, and mutual understanding have been credited as key to success of similar interventions for people with chronic disease in group settings $[33,34]$. It is worth noting that social support can be felt within online environments, yet this was not a theme that emerged in the current study. The lack of mention that participants felt supported, as others mentioned frequently in our previous study, could be a gap worth exploring further. The absence of feeling supported could have been a function of this specific population's value on support. Perhaps they do not value support so did not mention it as a benefit, or they really did not feel well-supported using the program. This was despite the addition of research assistants for coaching that was not offered in our previous trial of the CPMP [8].

It is also possible that the lack of perceived support limited participants' ability to fully engage and complete the program. Potentially, we can link an absence of notable support to the identified themes of feeling overwhelmed and ease of use. While we did offer individual or group training sessions for participants who desired more assistance with the CPMP, none attended group sessions. Attendance for in-person individual help sessions was irregular and the offered help was not universally used. Several participants suggested in their comments that pain self-management program orientation meetings should be required or included as a condition of their addiction treatment clinic program. The recognition that our participants desired or required more inperson help should be considered in future online program implementation. Feeling "overwhelmed" and improving ease of use could potentially be remediated by adding more in-person support. In other studies of online programs, support has been successfully delivered either in person, or via phone or text $[8,35]$. It is worth noting that for at least some of our participants, they required the use of clinic computers to participate in the CPMP. While much of the world now uses the Internet at least occasionally or owns a smartphone [36], our population in SUD did not all have easy access to technology. Some were homeless, in transitional housing, and/or had low cost phones that were not connected to the Internet and not always reliable.

Unique to our study, we specifically recruited adults who were in SUD treatment and who also identified that they had persistent pain. Our secondary aim investigated relationships between clinical factors and program engagement and found that both the percentage of reported pain relief in the last 24 hours and the use of alcohol for pain relief were positively associated with online program engagement. While correlation does not indicate causation, it is conceivable that those with better pain relief find it easier to engage in the online content. And while many substances were reportedly being used by this sample in an attempt to find pain relief, the use of alcohol was less frequently used and did not correlate significantly with program engagement. Conversely, having a positive drug screen indicating illicit substance use during the study period was related to less online program engagement. Therefore, it may be worth exploring whether the program is better suited for those people who are more stable in their substance 
use recovery and/or have immediate pain symptoms under better control when initiating program use.

Our descriptive approach guided by the theoretical framework of the IFSMT allowed us to identify specific variables unique to our participants' perspectives that can be explored in future intervention trials. For example, we recommend that a variety of instruction and support strategies be developed and tested for efficacy within this particular population to ensure the presence of structured self-management support and education. Online programs could be personalized by adding remedial training sessions for those who find computer programs daunting. Novel strategies can be tested for effects on proximal and distal outcomes as the IFSMT recommends. How selfmanagement can be applied to both pain and substance use issues simultaneously should be explored further. Including behavior change theories in future program development and testing might lead to more sustained desired changes.

Limitations of the study include the inability to question or clarify participant remarks further. The data provided were collected online and those who prefer to use verbal rather than written communications may not have fully participated. Also, the perspective of those who did not complete the study were not included. Therefore, a potential for response bias exists. Our data on substance use for pain relief was not captured with well-validated instruments due to a lack of testing on those specific items. Some of the text comments were brief and difficult to understand due to spelling and grammar mistakes. Focus groups or in-person interviews might have allowed for more detailed input from participants. However, due to the sensitive nature of substance use treatment, we felt online surveys were the most appropriate and least intrusive way to collect relevant data and preserve privacy. The sample was restricted to residents in the northwestern region of the U.S. so may not apply to other cultures or settings. Respondents were predominantly Caucasian. Yet, the population in MAT is often difficult to study due to challenges in living situations and symptoms. We consider it a study strength that we were able to collect enough data to formulate potential directions for future research. More information from those who did not complete the study would be helpful to better understand barriers to engagement. Nonetheless, the participants' words are perspectives that are often inaccessible due to the stigmatization of both addiction and chronic pain. The inquiry presented here allowed a glimpse into the lives of those who deal with an intrusive set of symptoms every day [10], and afforded us an opportunity to discover potential remedies.

\section{Conclusion}

The identified themes from this mixed method study suggest specific areas that can be targeted for developing and improving online self-management interventions for people with co-morbid pain and SUD. Frustrations in using E-health may be reduced by providing more upfront and ongoing support options tailored to meet individual needs. E-health program developers should consider users' needs for a simple, easily navigable human-computer interface. Our participants' experiences offer hope that gains can occur in knowledge and behavior change after exposure to online pain self-management concepts, even when usability is less than desired.

Future research is needed to examine more rigorously how clinical variables influence online program use. Pain symptom burdens and recovery treatment progress (e.g. reduced illicit drug use) may be important factors to address and stabilize before attempting self-management program engagement within populations in treatment for SUD. A variety of supportive structures and enrollment protocols can be trialed for greater online program use. Going forward, prospective randomized controlled studies can build on our findings by examining how and whether improving engagement with online pain selfmanagement reduces poorly managed pain symptoms and if this, in turn, can maximize SUD recovery outcomes. Such innovation may bring light to the under-appreciated role of pain in the opioid crisis, thereby reducing the tragic consequences of opioid misuse, overdose, and death.

\section{References}

[1] Center for Behavioral Health Statistics and Quality (2016), "Key Substance Use and Mental Health Indicators in the United States: Results from the 2015 National Survey on Drug Use and Health (HHS Publication No. SMA 16-4984, NSDUH Series H-51), http://www.samhsa.gov/data/, Accessed June 4, 2017.

[2] Centers for Disease Control and Prevention. "Prescription Opioid Overdose Data," August 1, 2017, https://www.cdc.gov/drugoverdose/data/overdose.html, Accessed May 28, 2018.

[3] C. Blanco, M.M. Wall, M. Okuda, S. Wang, M. Iza, M. Olfson, "Pain as a Predictor of Opioid Use Disorder in a Nationally Representative Sample", American Journal of Psychiatry, 2016, 173(12), pp. 1189-1195.

doi:10.1176/appi.ajp.2016.15091179

[4] J. Kennedy, J.M. Roll, T. Schraudner, S. Murphy, and S. McPherson, "Prevalence of Persistent Pain in the U.S. Adult Population: New Data from the 2010 National Health 
Interview Survey", Journal of Pain, 2014, 15(10), pp. 979984.

[5] K.E. Dunn, R.K. Brooner, M.R. Clark, "Severity and Interference of Chronic Pain in Methadone-maintained outpatients", Pain Medicine, 2014. doi: 10.1111/pme. 12430.

[6] R. Chou, G. Fanciullo, P. Fine, J. Adler, J. Ballantyne, P. Davies,...American Pain Society-American Academy of Pain Medicine Opioids Guidelines Panel. Clinical Guidelines for the use of Chronic Opioid Therapy in Chronic Noncancer Pain, The Journal of Pain, 2009, 10(2), pp.11330.

[7] L. Ruehlman, P. Karoly, and C. Enders, "A Randomized Controlled Evaluation of an Online Chronic Pain SelfManagement Program”, Pain, 2012, 153(2) doi:10.1016/j.pain.2011.10.025

[8] M. Wilson, J.M. Roll, C. Corbett, and C. Barbosa-Leiker, "Empowering Patients with Persistent Pain using an Internet-based Self-Management Program", Pain Management Nursing, 2015, 16(4), pp. 503-14. doi: 10.1016/j.pmn.2014.09.009; PMID:26088940

[9] M. Wilson, M. Roberts, and N. Sherazi, "Receptivity to an Online Pain Self-management Program among People with Persistent Pain in Opioid Addiction Treatment [abstract], The Journal of Pain, 18(4), S1-S162.

[10] M. Wilson, M. Finlay, M. Orr, C. Barbosa-Leiker, N. Sherazi, MLA Roberts, M. Layton, J.M. Roll, "Engagement in Online Pain Self-management Improves Pain in Adults on Medication-assisted Treatment for Opioid Use Disorders", Addictive Behaviors. Epub ahead of print Apr., 27, 2018. https://doi.org/10.1016/j.addbeh.2018.04.019

[11] R.L. Nahin, "Estimates of Pain Prevalence and Severity in Adults: United States, 2012”, Journal of Pain, 2015, 16(8), pp. 769-780.

[12] R. Knoerl, E.M. Lavoie Smith, and J. Weisberg, "Chronic Pain and Cognitive Behavioral Therapy: An Integrative Review", Western Journal of Nursing Research, 2016, 38(5), pp. 596-628. doi: 10.1177/0193945915615869

[13] D.D. Macea, K. Gajos, Y.A. Calil, and F. Fregni, “The Efficacy of Web-based Cognitive Behavioral Interventions for Chronic Pain: A Systematic and Meta-analysis", The Journal of Pain, 2010, 11(10), pp. 917-929. doi:10.1016/j/jpain.2010.06.005

[14] C. Eccleston, “Can 'Ehealth' Technology Deliver on its Promise of Pain Management for All?”, Pain, 2011, 152(8), pp. 1701-1702.

[15] Institute of Medicine Committee on Advancing Pain, Research, Care, and Education, "Relieving Pain in America: A Blueprint for Transforming Prevention, Care, Education, and Research", National Academies Press, Washington, D.C., 2011.

[16] Substance Abuse and Mental Health Services Administration, "Results from the 2012 National Survey on Drug Use and Health: Summary of National Findings", 2013, NSDUH Series H-46, HHS Publication No. (SMA) 13-4795. Rockville, MD.

[17] Centers for Disease Control and Prevention, "Vital Signs: Risk for Overdose from Methadone used for Pain Relief - United States, 1999-2010, MMWR Morb Mortal Wkly Rep, 2012, 61(26), pp. 493-497.

[18] L. Manchikanti, S. Abdi, S. Atluri, C.C Balog, R.M. Benyamin, M.V. Boswell,...American Society of Interventional Pain Physicians, "American Society of Interventional Pain Physicians (ASIPP) Guidelines for Responsible Opioid Prescribing in Chronic Non-cancer Pain: Part I--Evidence Assessment”, Pain Physician, 2012, 15(3 Suppl), pp. S1-65.

[19] E.O. Dumas, and G.M. Pollack, "Opioid Tolerance Development: A Pharmacokinetic/Pharmacodynamic Perspective", The AAPS Journal, 2008, 10(4), pp. 537-551. doi:10.1208/s12248-008-9056-1

[20] Department of Health and Human Services (DHHS). "National Pain Strategy", March 18, 2016, https://iprcc.nih.gov/National_Pain_Strategy/NPS_Main.ht m, Accessed March 22, 2016.

[21] E.C. Eyler, Chronic and Acute Pain and Pain Management for Patients in Methadone Maintenance Treatment, Am J Addict, 2013, 22(1), pp. 75-83. doi: 10.1111/j.1521-0391.2013.00308.x

[22] K. Lorig, P. Ritter, D. Laurent, and K. Plant, "The Internet-based Arthritis Self-management Program: A Oneyear Randomized Trial for Patients with Arthritis or Fibromyalgia", Arthritis \& Rheumatism, 2008, 59(7), pp. 1009-1017.

[23] R.M. Kadden, M.D. Litt, "The Role of Self-efficacy in the Treatment of Substance Use Disorders. Addict Behav, 2011, 36(12), pp. 1120-6. doi: 10.1016 /j.addbeh. 2011.07. 032. Epub 2011 Jul 29.

[24] M. Sandelowski, "What's in a Name? Qualitative Description Revisited," Research in Nursing \& Health, 2010, 33, pp. 77-84.

[25] M. Sandelowski, "Focus on Research Methods Whatever Happened to Qualitative Description?" Research in Nursing \& Health, 2000, 23, pp. 334-340.

[26] C. Cleeland, "The Brief Pain Inventory User Guide", The University of Texas MD Anderson Cancer Center. https://www.mdanderson.org/research/departments-labsinstitutes/departments-divisions/symptomresearch/symptom-assessment-tools.html. Published 2009. 
[27] P. Ryan, and K. Sawin, "The Individual and Family Self-Management Theory: Background and Perspectives on Context, Process, and Outcomes", Nursing Outlook, 2009, 57, pp. 217-225.

[28] M. Schreier. Qualitative Content Analysis in Practice, Sage, Thousand Oaks, CA, 2012

[29] M. Wilson, M. Shaw, "Participant perspectives on benefits and challenges of engaging in an online pain selfmanagement program, International Journal of Healthcare Information Systems and Informatics, 2017, 12(4).

[30] O.B. Kristjansdottir, E.A. Fors, A. Finset, S. Dulmen, S.H. Wigers, and H. Eide, "Written Online Situational Feedback via Mobile Phone to Support Self-management of Chronic Widespread Pain: A Usability Study of a Webbased Intervention", BioMed Central Musculoskeletal Disorders, 2011, 12(51), http://www. biomedcentral.com/ 1471-2474/12/51, Accessed June 1. 2016.

[31] V. Kruijssen, A. Staa, J. Dwarswaard, J. Veen, B. Mennema, and S. Adams, "Use of Online Self-Management Diaries in Asthma and COPD: A Qualitative Study of Subjects' and Professionals' Perceptions and Behaviors", Respiratory Care, 2015 60(8), pp. 1146-1156. doi: 10.4187/respcare.03795

[32] K. Trudea, L. Pujol, P. DasMahapatra, R. Wall, R. Black, and K. Zacharoff, "A Randomized Controlled Trial of an Online Self-management Program for Adults with Arthritis Pain", Journal of Behavioral Medicine, 2015, 38, pp. 483-496. doi: 10.1007/s10865-015-9622-9

[33] L.N. Andersen, M. Kohberg, L.G. Herborg, K. Søgaard, and K.K. Roessler, "“"Here We're All in the Same Boat"-- A Qualitative Study of Group Based Rehabilitation for Sicklisted Citizens with Chronic Pain", Scandinavian Journal of Psychology, 2014, 55(4), pp. 333-42, doi: 10.1111/sjop. 12121 .

[34] J. Barlow, R. Edwards, and A. Turner, "The Experience of Attending a Lay-led, Chronic Disease Self-management Programme from the Perspective of Participants with Multiple Sclerosis", Psychological Health, 2009, 24(10), pp. 1167-80, doi: 10.1080/08870440802040277

[35] M. Wilson, C. Hewes, C. Barbosa-Leiker, A. Mason, K. A. Wuestney, J.A. Shuen, M.P. Wilson. (2017). "Engaging adults with chronic disease in online depressive symptom self-management", Western Journal of Nursing Research. Epub ahead of print January 30, 2017.

[36] Pew Research Center, "Smartphone Ownership and Internet Usage Continues to Climb in Emerging Economies", http://www.pewglobal.org/2016/02/22/smart phone-ownership-and-internet-usage-continues-to-climbin-emerging-economies/, Accessed August 21, 2016 